\title{
Early-Onset Alzheimer's Disease: What Is Missing in Research?
}

\author{
Temitope Ayodele ${ }^{1,2,3}$ (D) - Ekaterina Rogaeva ${ }^{4}$ (I) $\cdot$ Jiji T. Kurup $^{1}$ (D) $\cdot$ Gary Beecham $^{5}$ (D) $\cdot$ Christiane Reitz $^{1,2,3,6}$
}

Accepted: 18 December 2020 / Published online: 19 January 2021

(C) The Author(s) 2021

\begin{abstract}
Purpose of Review Early-onset Alzheimer's disease (EOAD), defined as Alzheimer's disease (AD) occurring before age 65, is significantly less well studied than the late-onset form (LOAD) despite EOAD often presenting with a more aggressive disease progression. The aim of this review is to summarize the current understanding of the etiology of EOAD, their translation into clinical practice, and to suggest steps to be taken to move our understanding forward.

Recent Findings EOAD cases make up 5-10\% of AD cases but only 10-15\% of these cases show known mutations in the APP, $P S E N 1$, and PSEN2, which are linked to EOAD. New data suggests that these unexplained cases following a non-Mendelian pattern of inheritance is potentially caused by a mix of common and newly discovered rare variants. However, only a fraction of this genetic variation has been identified to date leaving the molecular mechanisms underlying this type of AD and their association with clinical, biomarker, and neuropathological changes unclear.

Summary While great advancements have been made in characterizing EOAD, much work is needed to disentangle the molecular mechanisms underlying this type of $\mathrm{AD}$ and to identify putative targets for more precise disease screening, diagnosis, prevention, and treatment.
\end{abstract}

Keywords Early-onset Alzheimer's disease $\cdot$ Epidemiology $\cdot$ Genetics $\cdot$ Biomarkers $\cdot$ Neuropathology

\section{Introduction}

Dementia is a term that is used to describe a category of diseases characterized by a decline in cognitive function that impairs daily function. To date, there are 50 million people that are living with dementia worldwide [1]. Alzheimer's disease (AD), the most common form of dementia, accounts for $50-75 \%$ of dementia cases [2]. Age is considered to be a principal risk factor for $\mathrm{AD}$, and it is used as a categorical marker [3,4]. The two main disease subcategories include early onset (EOAD) and late onset (LOAD) of AD, based on an arbitrary cut-off set at the age when individuals start to present symptoms, typically 65 years old [3, 4]. However, a cut-off of 60 years is sometimes used reflecting the arbitrary nature underlying this categorization [5]. Among reported AD cases, EOAD accounts for 5-10\% [6]. LOAD has a prevalence of $\sim 3.9 \%$ worldwide and an estimated annual incidence of $\sim 7.5$ per 1000 individuals above the age of 60 [7]. In comparison, data on prevalence and incidence of EOAD are much more limited. The studies that have assessed EOAD

This article is part of the Topical Collection on Dementia

Christiane Reitz

cr2101@cumc.columbia.edu

1 The Taub Institute for Research on Alzheimer's Disease and the Aging Brain, Columbia University, New York, NY, USA

2 The Gertrude H. Sergievsky Center, Columbia University, New York, NY, USA

3 Department of Neurology, Columbia University, New York, NY, USA
4 Tanz Centre for Research in Neurodegenerative Disease, University of Toronto, 60 Leonard Avenue, Toronto, ON M5T 0S8, Canada

5 The John P. Hussman Institute for Human Genomics, University of Miami, Miami, FL, USA

6 Department of Epidemiology, Sergievsky Center, Taub Institute for Research on the Aging Brain, Columbia University, 630 W 168th Street, New York, NY 10032, USA 
show that the annual prevalence and incidence of people between the age of 45 and 64 are approximately 24.2/100,000 and $6.3 / 100,000$, respectively $[8,9]$.

\section{Clinical Manifestations of EOAD}

In general, the clinical manifestation of $\mathrm{AD}$ is characterized by a predominant impairment of anterograde episodic memory. This symptom is typically accompanied by a multitude of cognitive impairments in domains, such as visuospatial, language, and executive function [7]. The combination of the aforementioned characteristics contributes to a global cognitive decline, eventually leading to a total dependent state, and death [10]. Although this typical clinical presentation of memory-predominant phenotypes overlaps between LOAD and EOAD cases, a subset of EOAD cases show an atypical presentation of preserved episodic memory function but focal cortical symptoms relating to language, visuospatial, or executive function [11]. In $25 \%$ of EOAD cases, there is a distinct phenotype of non-memory symptoms, in particular apraxia, visual dysfunction, fluent or non-fluent aphasia, executive dysfunction, or dyscalculia, that is seen as the disease progresses [11-13]. In addition, individuals with EOAD often present with a more aggressive disease progression and a shorter relative survival time [12], with the rate of progression driven at least in part by the nature of the underlying causative variant (see below). EOAD cases have a greater pathological burden (neuritic plaques and neurofibrillary tangles) compared to LOAD [14].

There have been no systematic large-scale studies assessing to what extent the different thresholds used affect estimates of prevalence, incidence, or clinical, genetic, and neuropathological variability. There is significant variability in age of onset both within and across families, which is partly explained by known genetic, environmental, or stochastic factors [15]. While some autosomal dominant cases develop first symptoms as early as their late 20 s, others develop the disease in their early 60 s (prevalence increases with age) $[8,9]$. Finally, in a recent study of mutation carriers and non-carrier in the Dominantly Inherited Alzheimer Network (DIAN) cohort, an observational study of families with PSEN1, PSEN2 or APP causal AD mutations, the personality traits of neuroticism, and conscientiousness were correlated with years to symptom onset, markers of tau pathology in the cerebrospinal fluid (CSF), and longitudinal rates of cognitive decline [16]. This suggests that there is clinically significant variability that needs to be thoroughly investigated in order to better characterize the disease.

\section{Neuropathological Changes}

The neuropathology of AD brains is characterized by extracellular accumulation of amyloid plaques, consisting of $A \beta 40$ and $A \beta 42$ peptides generated by the cleavage of APP, and intra-neuronal deposition of neurofibrillary tangles (NTF) composed of hyperphosphorylated tau protein (p-tau) [17]. These pathological hallmarks are often complemented by additional morphological changes including synaptic loss [18], neuronal loss [19], microglial activation [20], reactive astrocytes [21], neurovascular dysfunction [22], disruption of the blood brain barrier [23], and brain atrophy [24]. Up to $50 \%$ of AD patients exhibit concurrent $\alpha$-synuclein ( $\alpha$ Syn) pathology [25].

Both $A \beta$ and NFT neuropathological changes in both EOAD and LOAD progressively involve brain regions and neuronal cell types following a characteristic pattern [26]. $\mathrm{A} \beta$ accumulates initially across neocortical regions, then the limbic system, diencephalon, basal forebrain, and lastly the cerebellum. In contrast, tau involves first the entorhinal cortex and hippocampus, resulting in early-stage neurofibrillary degeneration, synaptic and neuronal loss and regional atrophy, and subsequently (early/mid stage) the locus coeruleus, basal forebrain, and associated regions of the neocortex, followed by the primary sensory cortex.

The extent to which the neuropathology underlying EOAD differs from that of LOAD remains unclear. Some distinctions have been reported. Temporoparietalprecuneus atrophy is seen in both EOAD and LOAD cases, but EOAD cases are seen to have a higher burden of neuritic plaques and NFT in these regions as well as the frontal cortex (albeit to a lesser degree) when compared to LOAD patients [26]. The neocortex, particularly parietal and occipitoparietal regions, seems to experience a similar burden, while the hippocampus is more likely to be spared in EOAD [27, 28]. Finally, individuals who develop AD at a younger age more often than not seem to have "purer" AD pathology with less concomitant neuropathological changes than older AD patients who often show a plethora of pathologies besides inclusions typical for AD (i.e., Lewy bodies, TDP-43, vascular pathology) [29-31]. It is important to recognize that many of the neuropathological studies conducted have accounted for age of onset and/or cognitive function but not duration of disease.

There are current research efforts to synchronize and fill the gap of understanding of the neuropathological overlap between LOAD and autosomal dominant EOAD by implementing a uniform neuropathological assessment protocol [32]. While this effort will be highly valuable, systematic harmonization of brain tissue assessment from non-Mendelian EOAD cases accounting for the majority of EOAD cases [33] in a similar manner is lacking. 


\section{Genetics of EOAD}

While the late-onset form of AD has a heritability of between 70 and $80 \%[3,34]$, the heritability of EOAD is higher amounting to $92-100 \%$ [3]. This correlates with the observation that up to $60 \%$ of EOAD subjects have at least one affected first-degree relative [33, 35].

\section{Mendelian EOAD}

As described above, families affected by EOAD can either follow a Mendelian (M-EOAD) or non-Mendelian inheritance pattern (NM-EOAD). Mendelian EOAD is caused by variants in APP [36], PSEN1 [37], or PSEN2 [37, 38]. Known to date are close to 330 mutations (duplications and missense) in these three genes accounting for $10-15 \%$ (APP), 30-70\% (PSEN1), and $<5 \%$ (PSEN2) of M-EOAD cases, respectively (https://www.alzforum.org/alzgene). While identification of these genetic variations has largely enhanced our understanding of $\mathrm{AD}$ pathogenesis, they only explain 10 $15 \%$ of familial EOAD cases, leaving the vast proportion of cases unexplained [33, 39].

Mutations in all three genes affect the amyloidogenic pathway leading to increased generation and aggregation of $A \beta$ $[40,41]$. A $\beta$ peptides result from the cleavage of APP by $\beta$ and $\gamma$-secretases; PSEN1 and PSEN2 are components of the $\gamma$-secretase complex [42]. While most mutations in APP result in increased levels of $A \beta$ aggregation and production [43], a protective APP variant (Icelandic mutation Ala673Thr) decreases $A \beta$ levels by $40 \%$ [44] (Fig. 1). Of note, mutations in the same codon (Ala673Val) cause AD when inherited in a recessive fashion [45]. APP carriers tend to have a notable level of amyloid angiopathy in their brain, which can lead to cerebral hemorrhage and stroke (i.e., in carriers of $A P P$ duplications) $[46,47]$.

Unlike $A P P$ mutations that cluster at the $\mathrm{A} \beta$ sequence [43], PSEN1 and PSEN2 mutations are found to be widely distributed in these genes (https://www.alzforum.org/alzgene). The majority of them are missense variants and the rest are inframe deletions/insertions [48-50]. Although PSEN1 and
PSEN2 are highly similar in terms of their genomic sequences, structures, and function, PSEN1 mutations appear considerably more pathogenic than PSEN2 [51]. PSEN1-related disease can have an onset as early as the third decade, while symptom onset associated with PSEN2 mutations shows significant variability and can occur beyond age 65 [51]. Notably, the brain expression of PSEN2 is $\sim 10$-fold lower than that of PSEN1 (https://www. proteinatlas.org/), which could explain more severe disease in PSEN1 carriers.

\section{Non-Mendelian EOAD}

Only $10-15 \%$ of EOAD cases can be explained by known mutations in PSEN1, PSEN2, and APP [33], and a significant portion of unexplained EOAD cases do not show a Mendelian pattern of inheritance, which inherently means that there are other unidentified genetic variations and mechanisms at play. Nevertheless, large-scale genomic studies conducted to date have mostly ignored this form of EOAD. It is likely a complex genetic disease caused by a mix of both rare and common variants, as suggested by a heritability study that assessed the genetic contribution in NM-EOAD cases from 32 US Alzheimer's Disease Centers to identify the underlying mode of inheritance [3]. This notion is supported by a recent linkage study among multiplex Caribbean Hispanic families loaded for NM-EOAD [52].

Over the past 10 years, genetic and biological studies identified common and rare genetic variants in the Sortilin-related receptor (SORL1) gene that are linked to both LOAD and EOAD [53-60]. Functional SORL1 is involved in trafficking of APP protein and can decrease levels of $A \beta$ peptides in a multitude of ways, including $A \beta$ peptides being trafficked to a lysosome for degradation, APP being sent to the Golgi apparatus, and the slowing down of the release of APP from the Golgi [61]. SORL1 variants could increase the risk of AD by decreasing the affinity for APP at the cell surface [62], thus modifying the role in which SORL1 plays in intracellular trafficking of APP [59, 63]. Recent meta-analyses and genomewide association studies observed a $\sim 20-90 \%$ increase in risk
Fig. 1 Representative APP mutations causing early-onset Alzheimer's disease with or without hemorrhage or stroke (in red). All known pathogenic missense substitutions in APP are localized in or around the $A \beta$ domain encoded by exons 16 and 17. The protective APP variant (Ala673Thr) is indicated in green

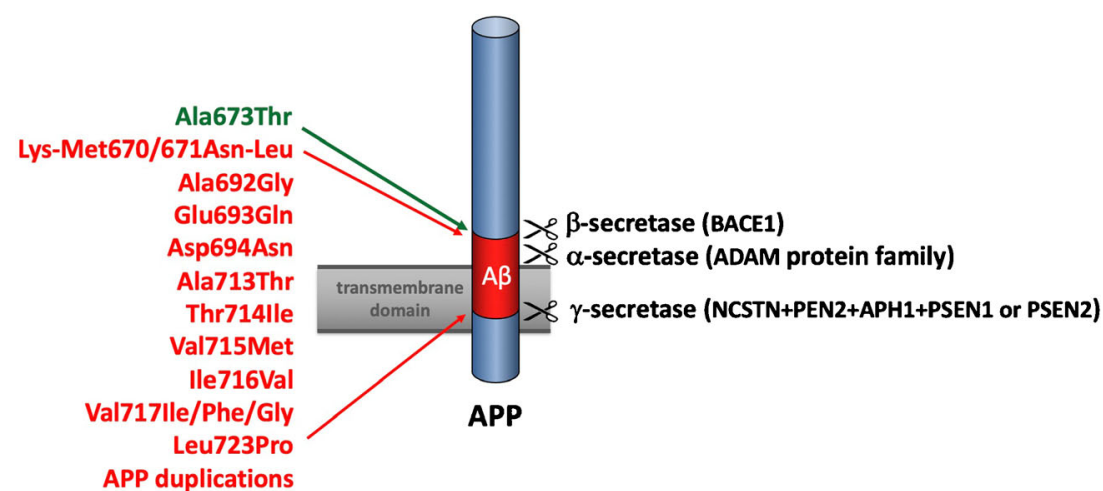


of LOAD [54, 64], and a five-fold increased risk of EOAD associated with rare SORL1 variants [55, 56], which is comparable to the effect size observed in APOE-e4 carriers [55, 56, 65]. A study that used the Exome Aggregation Consortium (ExAC) database (containing genetic variation of 60,706 human samples [66]) classified the pathogenicity of SORL1 variants and found that pathogenic SORL1 variants can increase $\mathrm{AD}$ risk by 12 -fold as well as cause an earlier age of onset (58.6 \pm 5.2 years) for SORL1 carriers [67]. Moreover, it was also found that protein-truncating variants of SORL1 are highly penetrant, which supports earlier findings that the loss of single copy of SORL1 is associated with AD [56]. Supporting the notion that these rare variants might explain part of the missing EOAD heritability, these rare protein-truncating SORL1 variants were observed exclusive in $\mathrm{AD}$ cases and absent in the ExAC database [67].

In addition, triggering receptor expressed on myeloid cells 2 (TREM2) has recently been reported to have a significant association with risk of EOAD. Recently, an exome-wide significant association between Arg47His TREM2 variant and EOAD risk was reported by study of the Alzheimer Disease Exome Sequencing-France dataset consisting of 927 LOAD cases, 852 EOAD cases, and 1273 controls [68]. Principal function of TREM2 is an intracellular adaptor for DNAX-activating protein of $12 \mathrm{kDa}$ (DAP12), as well as a modulator of myeloid cell number, proliferation, and survival $[68,69]$. Although the data is inconsistent, rare variants of TREM2 have been associated with $A \beta$ deposition, $A \beta$ uptake in microglia [70-72], increased tau in CSF, and the APOE pathway $[69,73]$. Together, these data suggest a strong connection to EOAD neuropathology, as well as both amyloid and tau hypotheses of AD [74].

Both genetic and environmental modifiers of AD risk could act through changes at $\mathrm{CpG}$ sites, which could affect DNA methylation (DNAm) levels. Similar to genetic mutations, changes in DNAm could lead to increased or decreased gene expression. Notably, CpGs are the most mutable sites in the genome, since methylated-cytosine can spontaneously change to thymine ( $\mathrm{C}>\mathrm{T}$ transition) [66]. Hence, combined genetic and DNAm studies are important for searching of disease modifiers [75].

Intriguingly, DNAm levels at some $\mathrm{CpGs}$ are age-related. The joint assessment of selected age-related CpGs is used in 15 reported DNAm clocks [76]. Each DNAm clock could reflect different aspects of biological aging, the pace of which could vary between different people, in contrast to the steady pace of chronological age. It is critical to investigate the expression patterns of genes containing age-related CpGs. Notably, among 1633 genes contributing to different DNAm clocks, 32 genes involved in the amyloid- $\beta$ pathway, including PSEN1 and BACE1, which encode $\gamma$-secretase and $\beta$ secretase, respectively [76].

Studies of DNAm clocks are very relevant to age-related disorders, such as AD. A reliable measure of biological age could be a factor in predicting $\mathrm{AD}$ onset in pre-symptomatic mutation carriers. The Horvath's DNAm clock, which assesses $\sim 350 \mathrm{CpGs}$, outperforms other clocks based on its multi-tissue applicability, including blood and brain [77]. DNAm-age acceleration (a discrepancy between DNAm-age and chronological age) was associated with several neurodegenerative disorders [78-81], including AD [82]. Importantly, DNAm-age acceleration was correlated with levels of $A \beta$ amyloid pathology in $\mathrm{AD}$ brains [83]. However, there is only one study of DNAm-age in familial AD. Recently, we investigated a family with identical triplets affected by LOAD in their late 70s, while one of the offspring developed EOAD at age 50. DNAm-age of the triplets was 6-10 years younger than chronological age. In contrast, DNAm-age was 9 years older in the offspring with EOAD, suggesting accelerated aging [84]. However, it is not clear if acceleration of DNAm-age causes aging or reacts to aging, which could be addressed in future longitudinal studies of DNAm clocks.

\section{Biofluid and Brain Imaging Biomarkers of EOAD}

To support the clinical diagnosis of EOAD in the clinical setting, identifying biochemical changes in CSF, blood or brain imaging are used as diagnostic tools as they can reflect the pathological changes associated with neurodegenerative diseases [85].

\section{CSF Biomarkers}

The most common biomarkers assessed to reflect AD pathology in biofluids are $\mathrm{A} \beta 40, \mathrm{~A} \beta 42$, total tau (T-tau), p-tau, and neurofilament light (NFL), an intraneuronal protein and constituent of the axonal cytoskeleton reflecting neuronal degeneration [85]. In CSF, p-tau is measured in order to represent the presence of tau pathology and NFT, whereas T-tau reflects neuronal injury or neurodegeneration [86], although there is evidence that CSF tau might rather represent an increase of neuronal secretion of tau in response to $A \beta$ pathology [87]. The $A \beta 42$ species of $A \beta$ peptides, which is released into CSF [88], is the earliest to accumulate and aggregate into both diffuse and core amyloid plaques [89]. The formation of these hydrophobic plaques consequently lowers $\mathrm{A} \beta 42$ that is available to be secreted, causing lower concentration levels of $\mathrm{A} \beta 42$ in CSF [85]. Based on the DIAN study, which-as described above - characterizes risk factors, pathophysiology, and biomarker changes across the disease course in carriers of autosomal dominant variants in the APP, PSEN1, or PSEN2 genes, $A \beta$ is the earliest detectable change to be observed in the neurodegenerative cascade 25 years before estimated age of onset [90]. Fibrillar amyloid demonstrated an annual mean percent change of $6.1 \%$, while there was a decreased rate of 
change of $-8.8 \%$ of CSF $\mathrm{A} \beta 42$ that slowed near the estimated age of onset [90]. Decline in precuneus metabolism measured by FDG PET took place 17 years before the estimated age of onset, followed by a decline in cognition and accelerated hippocampal atrophy taking place between 2 and 3 years before the estimated age of onset [90]. CSF p-tau is also seen to decline 3 years before the estimated age of onset in these longitudinal analyses, in contrast to previous observations from cross-sectional analyses that found that CSF tau and ptau levels are significantly higher 14 and 11 years before estimated age of onset, respectively [90]. Various other studies have confirmed that the decrease of CSF A $\beta 42$ and an increase of CSF tau and p-tau are diagnostically significant in AD dementia cases. A meta-analysis completed on 231 studies found a mean fold change of 0.56 (CSF A $\beta 42$ ), 2.54 (CSF T-tau), and 1.88 (CSF P-tau) in AD patients, respectively, which shows a stronger association of CSF T-tau and CSF P-tau biomarkers to AD than CSF A $\beta 42$ [91]. In contrast to this M-EOAD, there is a significant lack of studies assessing CSF biomarkers in NM-EOAD and potential differing patterns from autosomal dominant EOAD and LOAD. Clarifying longitudinal changes in these biomarkers from early adulthood and across the disease course will be critical to disentangle the molecular distinction of NM-EOAD from MEOAD and LOAD and improve the use of CSF-based biomarkers for screening and diagnosis in the clinical setting.

\section{Plasma Biomarkers}

Although measuring changes in CSF is advantageous because of its contact with the extracellular space of the brain, over the past two decades, significant efforts have been made to develop reliable, sensitive, and specific biomarkers of AD measured from blood as they are less invasive and significantly easier to obtain in both clinical and research settings [85]. While the close contact of CSF with the brain results in relatively high levels of molecules associated with brain disease, much lower amounts exist in the bloodstream. Additionally, plasma $A \beta$ can originate from other organs and tissues as well as be broken down by proteases, metabolized in the liver, or cleared by the kidneys [85, 92], all of which can influence the concentration of $\mathrm{A} \beta$ in the bloodstream. However, recent technological advancements in ultrasensitive immunoassays (such as the single-molecule array (Simoa technology) and mass spectrometry to assess plasma levels of molecules are providing promising results of the usefulness of these technologies for diagnosis, prognosis, and monitoring of progression of AD [93, 94].

Recent studies demonstrated that in particular the $A \beta 42 / 40$ ratio measured by ultrasensitive assays provides a sensitive assessment of amyloid burden that correlates both with the $\mathrm{A} \beta 42 / 40$ ratio in CSF and amyloid PET positivity in both M-EOAD and LOAD [95-98]. In addition, several studies have shown that NFL has diagnostic accuracy for AD similar to that of CSF NFL and increases over time as brain atrophy increases and cognition declines $[99,100]$. Studies in individuals with M-EOAD including the DIAN study further indicate that, similar to CSF, plasma NFL is elevated more than a decade prior to symptom onset, suggesting that it can serve as a sensitive diagnostic screening tool to identify individuals at risk [101]. While p-tau181 levels are elevated in AD, show associations with both $\mathrm{A} \beta$ and tau PET using agents such as $\mathrm{C}-\mathrm{PiB}(\mathrm{A} \beta)$ and ${ }^{18} \mathrm{~F}-\mathrm{AV}-1451(\mathrm{tau})$ (previously known as T807) [102-104], suggesting greater specificity for AD pathology than other tau species [105], overall the correlation of tau species measured from blood with CSF tau is relatively weak, potentially limiting the usefulness of currently available tau assays for screening, diagnosis, and monitoring of disease progression.

While it is reasonable to postulate that the abovementioned blood-based biomarker changes might behave similarly in NM-EOAD, there is a lack of studies assessing the prognostic and diagnostic validity of these measures in this type of AD.

\section{Neuroimaging}

Neuroimaging techniques done in both clinical and AD research settings include structural magnetic resonance imaging (MRI) and fluorodeoxyglucose (FDG)-positron emission tomography (PET), although the latter is often not covered by insurance and thus not performed. Structural MRIs assess the volumetric atrophy seen in AD, while FDG-PET identifies altered glucose metabolism. Current data on M-EOAD continues to parallel the typical presentation of glucose hypometabolism, amyloid deposition, and atrophy that is also seen across many cortical regions (thalamus, putamen, pallidum, hippocampus, caudate, amygdala, and accumbens) in LOAD cases [106]. However, besides the fact that in MEOAD these changes are seen earlier, they are often more widespread affecting additional areas including the putamen and thalamus [107], and there is often substantial hypometabolism seen first in posterior cingulate/precuneus and lateral parietal regions before it extends to include the frontal and temporal cortices [106]. Data from the DIAN study suggests that carriers of causal mutations exhibit cortical glucose hypometabolism and cortical thinning in the medial and lateral parietal lobes 5-10 years before the estimated age of onset, which suggests that FDG-PET and MRI changes are contemporaneous [106]. Subcortical regions seem to show a differing pattern. While all subcortical gray matter regions exhibited elevated $\mathrm{PiB}$ uptake, only the hippocampus showed reduced glucose metabolism [106].

Imaging $\mathrm{AD}$ pathology also includes the use of amyloid imaging tracers such as Pittsburgh Compound-B (PiB), as well as three amyloid radiotracers Amyvid ${ }^{\mathrm{TM}}$ (florbetapir 
F18), Neuraceq ${ }^{\mathrm{TM}}$ (florbetaben F18) and Vizamyl ${ }^{\mathrm{TM}}$ (flutemetamol F18) and one tau ligand (Tauvid ${ }^{\mathrm{TM}}$; 18Fflortaucipir) that are now approved by the Food and Drug Administration (FDA) [108].

In line with the morphological and metabolic changes that are seen before the estimated age of onset, elevated PiB levels are observed in almost every cortical region in an evenly dispersed manner almost 15 years before estimated age of onset [106]. However, the pallidum and caudate are exceptions because there is an observed increase of PiB levels but no evidence of metabolic or volumetric changes, which demonstrates that not all regions follow typical biomarker ordering [106]. In company with the elevated levels of PiB, tau-PET imaging demonstrates an increased level of tau tracer binding in the neocortex, and when compared to sporadic LOAD the levels of both agents are higher in M-EOAD [109]. Correlations between the specific component of tauopathy and the observed increase in tau-PET signaling are still unclear.

In line with M-EOAD, NM-EOAD sporadic cases with no family history of $\mathrm{AD}$ when compared to LOAD also present with more atrophy [110], robust neocortical glucose hypometabolism [111], and increased tau-PET signaling $[112,113]$. Although imaging studies are limited, these findings match the neurodegeneration and tauopathy that are seen in brain autopsies and show a more aggressive disease progression at younger age of onset. These observations are also reflected in analyses of the Alzheimer's Disease Neuroimaging Initiative (ADNI) dataset (unrelated $>65$ year-old subjects) showing more severe changes and disease progression MRI and biofluid-based biomarkers in persons with younger compared to older onset [114].

\section{Conclusion}

While there have been important advances in $\mathrm{AD}$ research in the past decades in understanding the mechanisms underlying EOAD, a significant part of its etiology remains unclear, hampering more accurate diagnosis and more effective treatment in the clinical setting. While routine screening using wholeexome sequencing is now feasible in the clinical setting, its interpretation (and associated genetic counseling) is largely limited to known mutations in APP, PSEN1, PSEN2, GRN, and $M P T$, which only account for a small subset of EOAD cases before age 65 . Clarifying the genetic, molecular, and clinical distinction of unexplained non-Mendelian EOAD from M-EOAD and LOAD, its pattern of cognitive decline, biomarker, and neuropathological changes over the disease course and the genetic and molecular underpinnings of its observed clinical variability will be critical to improve both diagnostic screening as well as developing more effective preventive and therapeutic targets. To do this, cohorts of NM-
EOAD cases with sufficient sample size, deep phenotypic characterization, longitudinal follow-up, comprehensive AD biomarker assessment, and an array of integrative data (i.e., genomic, epigenetic, proteomic, transcriptomic and metabolic data) are needed. Ideally, these datasets would comprise both family-based and case-control cohorts of various ancestral backgrounds to disentangle genetic variation specific to ethnic group, familial aggregation, or sporadic occurrence. Nextgeneration sequencing data coupled with other multi-omics approaches and extensive bioinformatics in such datasets would further allow to identify allelic effectors of identified loci, molecular underpinnings of disease etiology, progression and variation in age at onset, and the delineation between clinical endophenotypes. Identification of molecular disease signatures and pattern(s) of altered protein expression would allow to discriminate early disease stages, improve disease screening and diagnostics, identify subjects at risk for disease or more severe progression, and facilitate the identification of "druggable" molecular targets and successful repositioning of drugs for clinical application. Application of standardized assessment and analysis protocols comparable to those of the ongoing major studies on M-EOAD and LOAD will be critical to allow for valid comparison of data with these forms of AD.

As described above, the ADNI/DIAN Neuropathology Core has implemented a uniform assessment protocol to evaluate the neuropathological overlap between M-EOAD and LOAD. Similar collections of NM-EOAD brain tissue with standardized neuropathological assessment are needed to comprehensively delineate neuropathological overlap of both these forms with sporadic and familial NM-EOAD. Given that the NM-EOAD is likely heterogeneous, genetic analyses should test a variety of inheritance patters including recessive and polygenic effects.

Finally, there is a critical need for more studies to authenticate the use of ultrasensitive blood-based biomarkers as a screening, diagnostic, and monitoring tool in EOAD subtypes. Current data show that in particular the A $\beta 42 / 40$ ratio and NFL measured from blood using new technologies show a high degree of specificity and sensitivity to detect $\mathrm{AD}$ in individual years before onset of symptoms and correlate with CSF and imaging changes over the disease course. Validation of course and pattern of blood-based biomarkers in EOAD subtypes, ideally informed by genetic background, would be highly valuable for both clinical and research settings.

In-depth characterization of the clinical, genetic, and neuropathological distinction of unexplained EOAD from MEOAD and LOAD is critical to fully understand the etiology of $\mathrm{AD}$, characterize determinants and modulators of risk and age at onset, refine prevalence and incidence, and develop more effective targets for screening, prevention, and treatment. 
Code Availability N/A.

Funding This work was in part supported by the National Institutes of Health (NIH) grants RF1AG054080 (CR, GB, ER, TA), R01AG064614 (CR, GB), U01AG052410 (CR), P50AG008702 (CR), U01AG052410 (GB), and RF1AG054074 (GB). Dr. Rogaeva was further supported by the Canadian Consortium on Neurodegeneration in Aging.

\section{Data Availability N/A.}

\section{Compliance with Ethical Standards}

Conflict of Interest Temitope Ayodele, Ekaterina Rogaeva, Jiji T. Kurup, Gary Beecham, and Christiane Reitz declare that they have no conflict of interest.

Human and Animal Rights and Informed Consent This article does not contain any studies with human or animal subjects performed by any of the authors.

Open Access This article is licensed under a Creative Commons Attribution 4.0 International License, which permits use, sharing, adaptation, distribution and reproduction in any medium or format, as long as you give appropriate credit to the original author(s) and the source, provide a link to the Creative Commons licence, and indicate if changes were made. The images or other third party material in this article are included in the article's Creative Commons licence, unless indicated otherwise in a credit line to the material. If material is not included in the article's Creative Commons licence and your intended use is not permitted by statutory regulation or exceeds the permitted use, you will need to obtain permission directly from the copyright holder. To view a copy of this licence, visit http://creativecommons.org/licenses/by/4.0/.

\section{References}

Papers of particular interest, published recently, have been highlighted as:

- Of importance

•. Of major importance

1. World Health Organization 201910 facts on dementia 2019 [.

2. Alzheimer's Association. 2019 Alzheimer's disease facts and figures. Alzheimers Dement. 2019;15(3):321-87.

3. Wingo TS, Lah JJ, Levey AI, Cutler DJ. Autosomal recessive causes likely in early-onset Alzheimer disease. Arch Neurol. 2012;69(1):59-64.

4. Barber IS, Braae A, Clement N, Patel T, Guetta-Baranes T, Brookes K, et al. Mutation analysis of sporadic early-onset Alzheimer's disease using the NeuroX array. Neurobiol Aging. 2017;49(215):e1-8.

5. Szigeti K, Doody RS. Should EOAD patients be included in clinical trials? Alzheimers Res Ther. 2011;3(1):4.

6. Alzheimer's A. Early-onset dementia: a national challenge, a future crisis. Washington, DC: Alzheimer's Association; 2006.

7. Reitz C, Brayne C, Mayeux R. Epidemiology of Alzheimer disease. Nat Rev Neurol. 2011;7(3):137-52.

8. Lambert MA, Bickel H, Prince M, Fratiglioni L, Von Strauss E, Frydecka D, et al. Estimating the burden of early onset dementia; systematic review of disease prevalence. Eur J Neurol. 2014;21(4):563-9.

9. Renvoize E, Hanson M, Dale M. Prevalence and causes of young onset dementia in an English health district. Int $\mathrm{J}$ Geriatr Psychiatry. 2011;26(1):106-7.

10. Lane CA, Hardy J, Schott JM. Alzheimer's disease. Eur J Neurol. 2018;25(1):59-70.

11. van der Flier WM, Pijnenburg YA, Fox NC, Scheltens P. Earlyonset versus late-onset Alzheimer's disease: the case of the missing APOE $\varepsilon 4$ allele. Lancet Neurol. 2011;10(3):280-8.

12. Bateman RJ, Aisen PS, De Strooper B, Fox NC, Lemere CA, Ringman JM, et al. Autosomal-dominant Alzheimer's disease: a review and proposal for the prevention of Alzheimer's disease. Alzheimers Res Ther. 2011;3(1):1.

13. Ryan NS, Rossor MN. Correlating familial Alzheimer's disease gene mutations with clinical phenotype. Biomark Med. 2010;4(1): 99-112.

14. Marshall GA, Fairbanks LA, Tekin S, Vinters HV, Cummings JL. Early-onset Alzheimer's disease is associated with greater pathologic burden. J Geriatr Psychiatry Neurol. 2007;20(1):29-33.

15. Ryman DC, Acosta-Baena N, Aisen PS, Bird T, Danek A, Fox $\mathrm{NC}$, et al. Symptom onset in autosomal dominant Alzheimer disease: a systematic review and meta-analysis. Neurology. 2014;83(3):253-60.

16. Aschenbrenner AJ, Petros J, McDade E, Wang G, Balota DA, Benzinger TL, et al. Relationships between big-five personality factors and Alzheimer's disease pathology in autosomal dominant Alzheimer's disease. Alzheimers Dement (Amst). 2020;12(1): e12038.

17. Serrano-Pozo A, Frosch MP, Masliah E, Hyman BT. Neuropathological alterations in Alzheimer disease. Cold Spring Harb Perspect Med. 2011;1(1):a006189.

18. Chen MK, Mecca AP, Naganawa M, Finnema SJ, Toyonaga T, Lin SF, et al. Assessing synaptic density in Alzheimer disease with synaptic vesicle glycoprotein $2 \mathrm{~A}$ positron emission tomographic imaging. JAMA Neurol. 2018;75(10):1215-24.

19. West MJ, Coleman PD, Flood DG, Troncoso JC. Differences in the pattern of hippocampal neuronal loss in normal ageing and Alzheimer's disease. Lancet. 1994;344(8925):769-72.

20. Keren-Shaul H, Spinrad A, Weiner A, Matcovitch-Natan O, DvirSzternfeld R, Ulland TK, et al. A unique microglia type associated with restricting development of Alzheimer's disease. Cell. 2017;169(7):1276-90e17.

21. Liddelow SA, Guttenplan KA, Clarke LE, Bennett FC, Bohlen CJ, Schirmer L, et al. Neurotoxic reactive astrocytes are induced by activated microglia. Nature. 2017;541(7638):481-7.

22. Kisler K, Nelson AR, Montagne A, Zlokovic BV. Cerebral blood flow regulation and neurovascular dysfunction in Alzheimer disease. Nat Rev Neurosci. 2017;18(7):419-34.

23. Nation DA, Sweeney MD, Montagne A, Sagare AP, D'Orazio LM, Pachicano M, et al. Blood-brain barrier breakdown is an early biomarker of human cognitive dysfunction. Nat Med. 2019;25(2): 270-6.

24. Pini L, Pievani M, Bocchetta M, Altomare D, Bosco P, Cavedo E, et al. Brain atrophy in Alzheimer's disease and aging. Ageing Res Rev. 2016;30:25-48.

25. Arai Y, Yamazaki M, Mori O, Muramatsu H, Asano G, Katayama Y. Alpha-synuclein-positive structures in cases with sporadic Alzheimer's disease: morphology and its relationship to tau aggregation. Brain Res. 2001;888(2):287-96.

26. Palasí A, Gutiérrez-Iglesias B, Alegret M, Pujadas F, Olabarrieta $M$, Liébana $D$, et al. Differentiated clinical presentation of early and late-onset Alzheimer's disease: is 65 years of age providing a reliable threshold? J Neurol. 2015;262(5):1238-46.

27. Squire LR, Genzel L, Wixted JT, Morris RG. Memory consolidation. Cold Spring Harb Perspect Biol. 2015;7(8):a021766. 
28. Murray ME, Graff-Radford NR, Ross OA, Petersen RC, Duara R, Dickson DW. Neuropathologically defined subtypes of Alzheimer's disease with distinct clinical characteristics: a retrospective study. Lancet Neurol. 2011;10(9):785-96.

29. Savva GM, Wharton SB, Ince PG, Forster G, Matthews FE, Brayne C. Age, neuropathology, and dementia. N Engl J Med. 2009;360(22):2302-9.

30. Middleton LE, Grinberg LT, Miller B, Kawas C, Yaffe K. Neuropathologic features associated with Alzheimer disease diagnosis: age matters. Neurology. 2011;77(19):1737-44.

31. Haroutunian V, Schnaider-Beeri M, Schmeidler J, Wysocki M, Purohit DP, Perl DP, et al. Role of the neuropathology of Alzheimer disease in dementia in the oldest-old. Arch Neurol. 2008;65(9):1211-7.

32. Cairns NJ, Perrin RJ, Franklin EE, Carter D, Vincent B, Xie M, et al. Neuropathologic assessment of participants in two multi-center longitudinal observational studies: the Alzheimer Disease Neuroimaging Initiative (ADNI) and the Dominantly Inherited Alzheimer Network (DIAN). Neuropathology. 2015;35(4):390 400

33. Campion D, Dumanchin C, Hannequin D, Dubois B, Belliard S, Puel M, et al. Early-onset autosomal dominant Alzheimer disease: prevalence, genetic heterogeneity, and mutation spectrum. Am J Hum Genet. 1999;65(3):664-70.

34. Gatz M, Reynolds CA, Fratiglioni L, Johansson B, Mortimer JA, Berg S, et al. Role of genes and environments for explaining Alzheimer disease. Arch Gen Psychiatry. 2006;63(2):168-74.

35. Jarmolowicz AI, Chen HY, Panegyres PK. The patterns of inheritance in early-onset dementia: Alzheimer's disease and frontotemporal dementia. Am J Alzheimers Dis Other Dement. 2015;30(3):299-306

36. Goate A, Chartier-Harlin MC, Mullan M, Brown J, Crawford F, Fidani L, et al. Segregation of a missense mutation in the amyloid precursor protein gene with familial Alzheimer's disease. Nature. 1991;349(6311):704-6.

37. Sherrington R, Rogaev EI, Liang Y, Rogaeva EA, Levesque G, Ikeda $\mathrm{M}$, et al. Cloning of a gene bearing missense mutations in early-onset familial Alzheimer's disease. Nature. 1995;375(6534): 754-60.

38. Levy-Lahad E, Wasco W, Poorkaj P, Romano DM, Oshima J, Pettingell WH, et al. Candidate gene for the chromosome 1 familial Alzheimer's disease locus. Science. 1995;269(5226):973-7.

39. Bates KA, Verdile G, Li QX, Ames D, Hudson P, Masters CL, et al. Clearance mechanisms of Alzheimer's amyloid-beta peptide: implications for therapeutic design and diagnostic tests. Mol Psychiatry. 2009;14(5):469-86.

40. Wolfe MS. The gamma-secretase complex: membrane-embedded proteolytic ensemble. Biochemistry. 2006;45(26):7931-9.

41. Selkoe DJ, Wolfe MS. Presenilin: running with scissors in the membrane. Cell. 2007;131(2):215-21.

42. Haass C, De Strooper B. The presenilins in Alzheimer's diseaseproteolysis holds the key. Science. 1999;286(5441):916-9.

43. Sisodia SS, St George-Hyslop PH. Gamma-Secretase, Notch, Abeta and Alzheimer's disease: where do the presenilins fit in? Nat Rev Neurosci. 2002;3(4):281-90.

44. Jonsson T, Atwal JK, Steinberg S, Snaedal J, Jonsson PV, Bjornsson $\mathrm{S}$, et al. A mutation in APP protects against Alzheimer's disease and age-related cognitive decline. Nature. 2012;488(7409):96-9.

45. Di Fede G, Catania M, Morbin M, Rossi G, Suardi S, Mazzoleni $\mathrm{G}$, et al. A recessive mutation in the APP gene with dominantnegative effect on amyloidogenesis. Science. 2009;323(5920): 1473-7.

46. Rovelet-Lecrux A, Hannequin D, Raux G, Le Meur N, Laquerrière A, Vital A, et al. APP locus duplication causes autosomal dominant early-onset Alzheimer disease with cerebral amyloid angiopathy. Nat Genet. 2006;38(1):24-6.

47. Guyant-Marechal I, Berger E, Laquerrière A, Rovelet-Lecrux A, Viennet G, Frebourg T, et al. Intrafamilial diversity of phenotype associated with app duplication. Neurology. 2008;71(23):1925-6.

48. Crook R, Verkkoniemi A, Perez-Tur J, Mehta N, Baker M, Houlden $\mathrm{H}$, et al. A variant of Alzheimer's disease with spastic paraparesis and unusual plaques due to deletion of exon 9 of presenilin 1. Nat Med. 1998;4(4):452-5.

49. Rogaeva EA, Fafel KC, Song YQ, Medeiros H, Sato C, Liang Y, et al. Screening for PS1 mutations in a referral-based series of AD cases: 21 novel mutations. Neurology. 2001;57(4):621-5.

50. De Jonghe C, Cruts M, Rogaeva EA, Tysoe C, Singleton A, Vanderstichele H, et al. Aberrant splicing in the presenilin-1 intron 4 mutation causes presenile Alzheimer's disease by increased Abeta42 secretion. Hum Mol Genet. 1999;8(8):1529-40.

51. Snider BJ, Norton J, Coats MA, Chakraverty S, Hou CE, Jervis R, et al. Novel presenilin 1 mutation $(\mathrm{S} 170 \mathrm{~F})$ causing Alzheimer disease with Lewy bodies in the third decade of life. Arch Neurol. 2005;62(12):1821-30.

52. Cheng R, Tang M, Martinez I, Ayodele T, Baez P, ReyesDumeyer D, et al. Linkage analysis of multiplex Caribbean Hispanic families loaded for unexplained early-onset cases identifies novel Alzheimer's disease loci. Alzheimers Dement (Amst). 2018;10:554-62.

53. Pottier C, Hannequin D, Coutant S, Rovelet-Lecrux A, Wallon D, Rousseau S, et al. High frequency of potentially pathogenic SORL1 mutations in autosomal dominant early-onset Alzheimer disease. Mol Psychiatry. 2012;17(9):875-9.

54. Vardarajan BN, Zhang Y, Lee JH, Cheng R, Bohm C, Ghani M, et al. Coding mutations in SORL1 and Alzheimer disease. Ann Neurol. 2015;77(2):215-27.

55. Nicolas G, Charbonnier C, Wallon D, Quenez O, Bellenguez C, Grenier-Boley B, et al. SORL1 rare variants: a major risk factor for familial early-onset Alzheimer's disease. Mol Psychiatry. 2016;21(6):831-6.

56. Verheijen J, Van den Bossche T, van der Zee J, Engelborghs S, Sanchez-Valle R, Lladó A, et al. A comprehensive study of the genetic impact of rare variants in SORL1 in European early-onset Alzheimer's disease. Acta Neuropathol. 2016;132(2):213-24.

57. Andersen OM, Rudolph IM, Willnow TE. Risk factor SORL1: from genetic association to functional validation in Alzheimer's disease. Acta Neuropathol. 2016;132(5):653-65.

58. Lambert JC, Ibrahim-Verbaas CA, Harold D, Naj AC, Sims R, Bellenguez C, et al. Meta-analysis of 74,046 individuals identifies 11 new susceptibility loci for Alzheimer's disease. Nat Genet. 2013;45(12):1452-8.

59. Rogaeva E, Meng Y, Lee JH, Gu Y, Kawarai T, Zou F, et al. The neuronal sortilin-related receptor SORL1 is genetically associated with Alzheimer disease. Nat Genet. 2007;39(2):168-77.

60. Reitz C, Cheng R, Rogaeva E, Lee JH, Tokuhiro S, Zou F, et al. Meta-analysis of the association between variants in SORL1 and Alzheimer disease. Arch Neurol. 2011;68(1):99-106.

61. Campion D, Charbonnier C, Nicolas G. SORL1 genetic variants and Alzheimer disease risk: a literature review and meta-analysis of sequencing data. Acta Neuropathol. 2019;138(2):173-86.

62. Cuccaro ML, Carney RM, Zhang Y, Bohm C, Kunkle BW, Vardarajan BN, et al. SORL1 mutations in early- and late-onset Alzheimer disease. Neurol Genet. 2016;2(6):e116.

63. Barthelson K, Newman M, Lardelli M. Sorting out the role of the sortilin-related receptor 1 in Alzheimer's disease. J Alzheimers Dis Rep. 2020;4(1):123-40.

64. Kunkle BW, Grenier-Boley B, Sims R, Bis JC, Damotte V, Naj $\mathrm{AC}$, et al. Genetic meta-analysis of diagnosed Alzheimer's disease identifies new risk loci and implicates $\mathrm{A} \beta$, tau, immunity and lipid processing. Nat Genet. 2019;51(3):414-30. 
65. Corder EH, Saunders AM, Strittmatter WJ, Schmechel DE, Gaskell PC, Small GW, et al. Gene dose of apolipoprotein E type 4 allele and the risk of Alzheimer's disease in late onset families. Science. 1993;261(5123):921-3.

66. Lek M, Karczewski KJ, Minikel EV, Samocha KE, Banks E, Fennell T, et al. Analysis of protein-coding genetic variation in 60,706 humans. Nature. 2016;536(7616):285-91.

67. Holstege H, van der Lee SJ, Hulsman M, Wong TH, van Rooij JG, Weiss M, et al. Characterization of pathogenic SORL1 genetic variants for association with Alzheimer's disease: a clinical interpretation strategy. Eur J Hum Genet. 2017;25(8):973-81.

68. Bellenguez C, Charbonnier C, Grenier-Boley B, Quenez O, Le Guennec K, Nicolas G, et al. Contribution to Alzheimer's disease risk of rare variants in TREM2, SORL1, and ABCA7 in 1779 cases and 1273 controls. Neurobiol Aging. 2017;59(220):e1-9.

69. Gratuze M, Leyns CEG, Holtzman DM. New insights into the role of TREM2 in Alzheimer's disease. Mol Neurodegener. 2018;13(1):66

70. Jiang T, Tan L, Zhu XC, Zhang QQ, Cao L, Tan MS, et al. Upregulation of TREM2 ameliorates neuropathology and rescues spatial cognitive impairment in a transgenic mouse model of Alzheimer's disease. Neuropsychopharmacology. 2014;39(13): 2949-62.

71. Ulrich JD, Finn MB, Wang Y, Shen A, Mahan TE, Jiang H, et al. Altered microglial response to Abeta plaques in APPPS1-21 mice heterozygous for TREM2. Mol Neurodegener. 2014;9:20.

72. Kleinberger G, Yamanishi Y, Suarez-Calvet M, Czirr E, Lohmann $\mathrm{E}$, Cuyvers E, et al. TREM2 mutations implicated in neurodegeneration impair cell surface transport and phagocytosis. Sci Transl Med. 2014;6(243):243ra86.

73. Lill CM, Rengmark A, Pihlstrøm L, Fogh I, Shatunov A, Sleiman $\mathrm{PM}$, et al. The role of TREM2 R47H as a risk factor for Alzheimer's disease, frontotemporal lobar degeneration, amyotrophic lateral sclerosis, and Parkinson's disease. Alzheimers Dement. 2015;11(12):1407-16.

74. Kametani F, Hasegawa M. Reconsideration of amyloid hypothesis and tau hypothesis in Alzheimer's disease. Front Neurosci. 2018;12:25.

75. Zhang M, Ferrari R, Tartaglia MC, Keith J, Surace EI, Wolf U, et al. A C6orf10/LOC101929163 locus is associated with age of onset in C9orf72 carriers. Brain. 2018;141(10):2895-907.

76. Bergsma T and Rogaeva E. DNA methylation clocks and their predictive capacity for ageing phenotypes and healthspan. Neuroscience Insights, 2020 (Submitted).

77. Horvath S. DNA methylation age of human tissues and cell types. Genome Biol. 2013;14(10):R115.

78. Horvath S, Ritz BR. Increased epigenetic age and granulocyte counts in the blood of Parkinson's disease patients. Aging. 2015;7(12):1130-42.

79. Horvath S, Langfelder P, Kwak S, Aaronson J, Rosinski J, Vogt $\mathrm{TF}$, et al. Huntington's disease accelerates epigenetic aging of human brain and disrupts DNA methylation levels. Aging (Albany NY). 2016;8(7):1485-512.

80. Zhang M, Tartaglia MC, Moreno D, Sato C, McKeever P, Weichert A, et al. DNA methylation age-acceleration is associated with disease duration and age at onset in C9orf72 patients. Acta Neuropathol. 2017;134:271-9.

81. Zhang M, McKeever PM, Xi Z, Moreno D, Sato C, Bergsma T, et al. DNA methylation age acceleration is associated with ALS age of onset and survival. Acta Neuropathol. 2020;139(5):943-6

82. Levine ME, Lu AT, Bennett DA, Horvath S. Epigenetic age of the pre-frontal cortex is associated with neuritic plaques, amyloid load, and Alzheimer's disease related cognitive functioning. Aging. 2015;7(12):1198-211.

83. Degerman S, Josefsson M, Nordin Adolfsson A, Wennstedt S, Landfors M, Haider Z, et al. Maintained memory in aging is associated with young epigenetic age. Neurobiol Aging. 2017;55:167-71.

84. Zhang M, Dilliott AA, Khallaf R, Robinson JF, Hegele RA, Comishen $\mathrm{M}$, et al. Genetic and epigenetic study of an Alzheimer's disease family with monozygotic triplets. Brain. 2019;142(11):3375-81.

85. Blennow K, Zetterberg H. Biomarkers for Alzheimer's disease: current status and prospects for the future. J Intern Med. 2018;284(6):643-63.

86. Jack CR Jr, Bennett DA, Blennow K, Carrillo MC, Feldman HH, Frisoni GB, et al. A/T/N: an unbiased descriptive classification scheme for Alzheimer disease biomarkers. Neurology. 2016;87(5):539-47.

87. Allen M, Kachadoorian M, Quicksall Z, Zou F, Chai HS, Younkin $\mathrm{C}$, et al. Association of MAPT haplotypes with Alzheimer's disease risk and MAPT brain gene expression levels. Alzheimers Res Ther. 2014;6(4):39.

88. Niemantsverdriet E, Valckx S, Bjerke M, Engelborghs S. Alzheimer's disease CSF biomarkers: clinical indications and rational use. Acta Neurol Belg. 2017;117(3):591-602.

89. Iwatsubo T, Odaka A, Suzuki N, Mizusawa H, Nukina N, Ihara Y. Visualization of a beta 42(43) and a beta 40 in senile plaques with end-specific a beta monoclonals: evidence that an initially deposited species is a beta 42(43). Neuron. 1994;13(1):45-53.

90. McDade E, Wang G, Gordon BA, Hassenstab J, Benzinger TLS, Buckles V, et al. Longitudinal cognitive and biomarker changes in dominantly inherited Alzheimer disease. Neurology. 2018;91(14): e1295-e306.

91. Olsson B, Lautner R, Andreasson U, Öhrfelt A, Portelius E, Bjerke $\mathrm{M}$, et al. CSF and blood biomarkers for the diagnosis of Alzheimer's disease: a systematic review and meta-analysis. Lancet Neurol. 2016;15(7):673-84.

92. Toledo JB, Shaw LM, Trojanowski JQ. Plasma amyloid beta measurements - a desired but elusive Alzheimer's disease biomarker. Alzheimers Res Ther. 2013;5(2):8.

93. Shi L, Baird AL, Westwood S, Hye A, Dobson R, Thambisetty M, et al. A decade of blood biomarkers for Alzheimer's disease research: an evolving field, improving study designs, and the challenge of replication. J Alzheimers Dis. 2018;62(3):1181-98.

94. Hampel H, Vergallo A, Afshar M, Akman-Anderson L, Arenas J, Benda N, et al. Blood-based systems biology biomarkers for nextgeneration clinical trials in Alzheimer's disease. Dialogues Clin Neurosci. 2019;21(2):177-91.

95. Ovod V, Ramsey KN, Mawuenyega KG, Bollinger JG, Hicks T, Schneider T, et al. Amyloid beta concentrations and stable isotope labeling kinetics of human plasma specific to central nervous system amyloidosis. Alzheimers Dement. 2017;13(8):841-9.

96. Nabers A, Perna L, Lange J, Mons U, Schartner J, Guldenhaupt J, et al. Amyloid blood biomarker detects Alzheimer's disease. EMBO Mol Med. 2018;10(5).

97. Palmqvist S, Janelidze S, Stomrud E, Zetterberg H, Karl J, Zink K, et al. Performance of fully automated plasma assays as screening tests for Alzheimer disease-related beta-amyloid status. JAMA Neurol. 2019.

98. Kaneko N, Nakamura A, Washimi Y, Kato T, Sakurai T, Arahata $\mathrm{Y}$, et al. Novel plasma biomarker surrogating cerebral amyloid deposition. Proc Jpn Acad Ser B Phys Biol Sci. 2014;90(9):35364.

99. Gisslén M, Price RW, Andreasson U, Norgren N, Nilsson S, Hagberg L, et al. Plasma concentration of the neurofilament light protein (NFL) is a biomarker of CNS injury in HIV infection: a cross-sectional study. EBioMedicine. 2016;3:135-40.

100. Mattsson N, Andreasson U, Zetterberg H, Blennow K. Alzheimer's disease neuroimaging I. Association of Plasma Neurofilament Light With Neurodegeneration in Patients With Alzheimer Disease. JAMA Neurol. 2017;74(5):557-66. 
101. Preische O, Schultz SA, Apel A, Kuhle J, Kaeser SA, Barro C, et al. Serum neurofilament dynamics predicts neurodegeneration and clinical progression in presymptomatic Alzheimer's disease. Nat Med. 2019;25(2):277-83.

102. Brier MR, Gordon B, Friedrichsen K, McCarthy J, Stern A, Christensen J, et al. Tau and A $\beta$ imaging, CSF measures, and cognition in Alzheimer's disease. Sci Transl Med. 338;2016(8): 338 ra66.

103. Chhatwal JP, Schultz AP, Marshall GA, Boot B, Gomez-Isla T, Dumurgier J, et al. Temporal T807 binding correlates with CSF tau and phospho-tau in normal elderly. Neurology. 2016;87(9): 920-6.

104. Gordon BA, Friedrichsen K, Brier M, Blazey T, Su Y, Christensen $\mathrm{J}$, et al. The relationship between cerebrospinal fluid markers of Alzheimer pathology and positron emission tomography tau imaging. Brain. 2016;139(Pt 8):2249-60.

105. Jack CR Jr, Bennett DA, Blennow K, Carrillo MC, Dunn B, Haeberlein SB, et al. NIA-AA research framework: toward a biological definition of Alzheimer's disease. Alzheimers Dement. 2018;14(4):535-62.

106. Benzinger TL, Blazey T, Jack CR Jr, Koeppe RA, Su Y, Xiong C, et al. Regional variability of imaging biomarkers in autosomal dominant Alzheimer's disease. Proc Natl Acad Sci U S A. 2013;110(47):E4502-9.

107. Knight WD, Okello AA, Ryan NS, Turkheimer FE, Rodriguez Martinez de Llano S, Edison P, et al. Carbon-11-Pittsburgh compound $\mathrm{B}$ positron emission tomography imaging of amyloid deposition in presenilin 1 mutation carriers. Brain. 2011;134(Pt 1): 293-300.

108. Rowley PA, Samsonov AA, Betthauser TJ, Pirasteh A, Johnson SC, Eisenmenger LB. Amyloid and Tau PET imaging of
Alzheimer disease and other neurodegenerative conditions. Seminars in Ultrasound, CT and MRI. 2020.

109. Gordon BA, Blazey TM, Christensen J, Dincer A, Flores S, Keefe $\mathrm{S}$, et al. Tau PET in autosomal dominant Alzheimer's disease: relationship with cognition, dementia and other biomarkers. Brain. 2019;142(4):1063-76.

110. Moller C, Vrenken H, Jiskoot L, Versteeg A, Barkhof F, Scheltens $\mathrm{P}$, et al. Different patterns of gray matter atrophy in early- and lateonset Alzheimer's disease. Neurobiol Aging. 2013;34(8):2014 22.

111. Rabinovici GD, Furst AJ, Alkalay A, Racine CA, O'Neil JP, Janabi $\mathrm{M}$, et al. Increased metabolic vulnerability in early-onset Alzheimer's disease is not related to amyloid burden. Brain. 2010;133(Pt 2):512-28.

112. Cho H, Choi JY, Lee SH, Lee JH, Choi YC, Ryu YH, et al. Excessive tau accumulation in the parieto-occipital cortex characterizes early-onset Alzheimer's disease. Neurobiol Aging. 2017;53:103-11.

113. Scholl M, Ossenkoppele R, Strandberg O, Palmqvist S, Fs SB, Jogi J, et al. Distinct 18F-AV-1451 tau PET retention patterns in early- and late-onset Alzheimer's disease. Brain. 2017;140(9): 2286-94.

114. Holland D, Desikan RS, Dale AM, LK ME. Alzheimer's Disease Neuroimaging I. Rates of decline in Alzheimer disease decrease with age. PLoS One. 2012;7(8):e42325.

Publisher's Note Springer Nature remains neutral with regard to jurisdictional claims in published maps and institutional affiliations. 